\title{
Traumatic Brain Injury: Care and Treatment of Operation Enduring Freedom and Operation Iraqi Freedom Veterans
}

\author{
Amalia K. Corby-Edwards
}

Analyst in Public Health and Epidemiology

November 25, 2009 


\section{Summary}

Traumatic brain injury (TBI), defined in the medical literature as a disruption in brain function that is caused by a head injury, has become known as one of the "signature wounds" of the wars in Iraq and Afghanistan due to its high occurrence in post-deployment servicemembers and veterans of these wars. As servicemembers return home, many need ongoing care for mild, moderate, or severe TBI. The growing number of TBI patients and the nature of their injuries creates the need for increased treatment capacity for veterans, and raises a number of policy issues that Congress may move to consider.

In the civilian population, traumatic brain injuries are mainly due to motor vehicle crashes, falls, assaults, and blows to the head. TBI severity may range from "mild," a brief change in mental status or consciousness after the injury, to "severe," an extended period of unconsciousness or amnesia. In addition to physical symptoms, mental health diagnoses such as post-traumatic stress disorder (PTSD), depression, and anxiety are common for TBI patients, as well as substance abuse. Due to the variable nature of TBI injury and recovery, there is not one standard of care or treatment regimen for TBI; patients' needs are diverse, depending on the severity of illness and the presence of co-conditions.

It has been estimated by a recent RAND study that as many as $20 \%$ of Operation Enduring Freedom and Operation Iraqi Freedom (OEF/OIF) veterans experience TBI. The Department of Veterans Affairs (VA) has screened almost 250,000 OEF/OIF veterans entering the Veterans Health Administration (VHA) system as of January 2009. As servicemembers return home, these numbers will increase.

VA provides a wide range of services to address the needs of veterans with TBI, including outreach, education, and benefits enrollment information. The FY2010 VA Budget included assurances that VA is working to fund programs that improve veterans' access to mental health services across the country, including those who suffer from TBI as a result of their service in OEF/OIF. In responding to this influx of veterans with TBI and other common OEF/OIF illnesses, policymakers and others have identified areas of concern, including challenges in screening, diagnosis, treatment, and access to care.

This report provides a review of TBI as an illness, its prevalence among veterans, current activity to address the issue in the Department of Veterans Affairs, and current policy issues. 


\section{Contents}

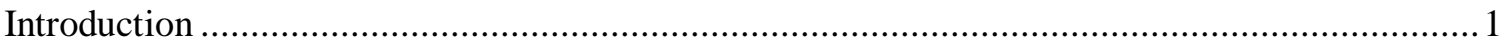

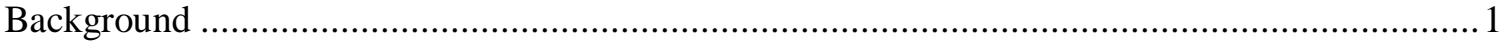

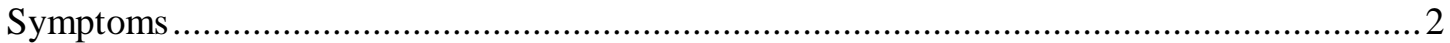

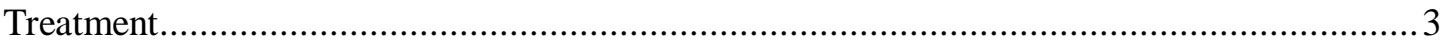

Care and Treatment of Veterans ............................................................................

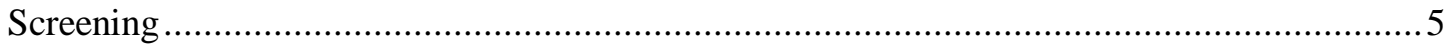

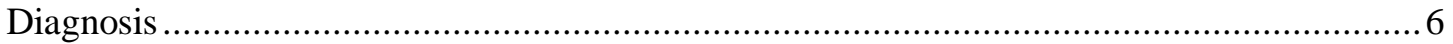

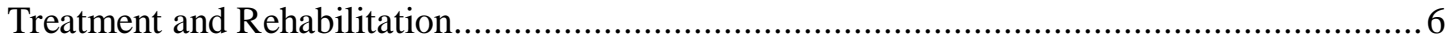

Polytrauma System of Care ................................................................... 7

Coordination of Treatment ..........................................................................9

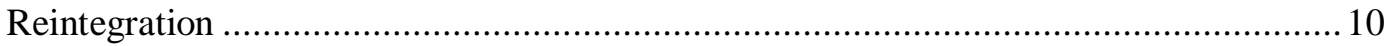

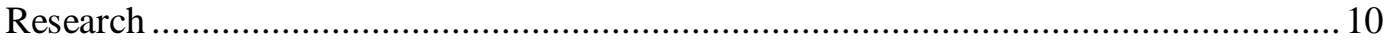

Access to Care ......................................................................................... 11

Physical, Financial, and Other Barriers to Accessing Care ...................................... 11

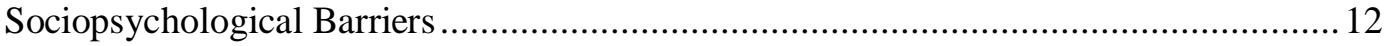

Issues for Congress ........................................................................... 13

Identification and Screening ................................................................ 13

Continuity of Care ..................................................................................... 14

Staffing and Access to Care...................................................................... 14

Social and Psychological Issues ................................................................ 14

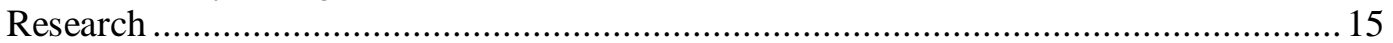

\section{Figures}

Figure 1. Causes of TBI in the Civilian Population, 2004...................................................2

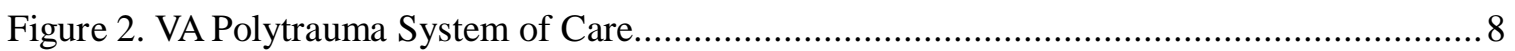

\section{Contacts}

Author Contact Information 


\section{Introduction}

Traumatic brain injury (TBI) has become known as one of the "signature injuries" of the wars in Iraq and Afghanistan due to its high occurrence in post-deployment servicemembers and veterans of these wars. ${ }^{1}$ Soldiers in Operation Iraqi Freedom (OIF) and Operation Enduring Freedom (OEF) are experiencing TBI in large numbers due to blast injuries caused by exposure to improvised explosive devices, rocket-propelled grenades, and mortar/artillery shells. ${ }^{2}$ According to one study, among active-duty servicemembers treated at Walter Reed Army Medical Center from 2003 to 2005 , nearly $30 \%$ of all combat-related injuries included a brain injury. ${ }^{3}$ Continuous improvements in battlefield medicine have increased soldiers' survival rates from these attacks. Consequently, increased survival rates necessitate long- and short-term treatment of larger numbers of wounded soldiers.

As servicemembers return home, many may need ongoing care for TBI and related conditions. The growing number of TBI patients and the nature of their injuries create the need for increased treatment capacity for veterans. In responding to these patients, the Department of Veterans' Affairs (VA), Department of Defense (DOD), Congress, and other entities have identified TBI screening, diagnosis, and treatment issues that need to be addressed, including accessibility of care, coordination of care between DOD and VA, and the simultaneous care of TBI co-conditions, notably, post-traumatic stress disorder (PTSD), another common OEF/OIF diagnosis. Congress has allocated significant resources toward TBI research, treatment, and care to address these issues.

This report will describe the characteristics of TBI and provide the reader with an overview of current VA activity and key policy issues.

\section{Background}

In medical literature, traumatic brain injury is defined as a disruption in brain function that is caused by a head injury. ${ }^{4}$ In the civilian population, these injuries are mainly due to motor vehicle crashes, falls, assaults, and blows to the head. Figure 1 shows this distribution. In servicemembers, TBI mainly results from blast injuries caused by exposure to improvised explosive devices, rocket-propelled grenades, land mines, and mortar/artillery shells, as well as motor vehicle crashes, falls, and assaults. ${ }^{5}$ The following sections provide an overview of the symptoms and treatment of traumatic brain injury.

\footnotetext{
${ }^{1}$ Operation Enduring Freedom (OEF) includes operations in Afghanistan and other Global War on Terror (GWOT) operations ranging from the Philippines to Djibouti that began immediately after the terrorist attacks on September 11, 2001, and continue today; Operation Iraqi Freedom (OIF) began in the fall of 2002 with the buildup of troops for the March 2003 invasion of Iraq and continues with counter-insurgency and stability operations.

${ }^{2}$ T. Tanielian, L. Jaycox, ed., Invisible Wounds of War: Psychological and Cognitive Injuries, Their Consequences, and Services to Assist Recovery, Rand, 2008.

${ }^{3}$ S. Okie, “Traumatic Brain Injury in the War Zone," New England Journal of Medicine, vol. 352, no. 20 (2005), pp. 2043-2046.

${ }^{4}$ T. Tanielian, L. Jaycox , ed., Invisible Wounds of War: Psychological and Cognitive Injuries, Their Consequences, and Services to Assist Recovery, Rand, 2008.

${ }^{5}$ Defense and Veterans Brain Injury Center: TBI and the Military, http://www.dvbic.org/TBI-The-Military.aspx (accessed September 14, 2009).
} 
Figure I. Causes of TBI in the Civilian Population, 2004

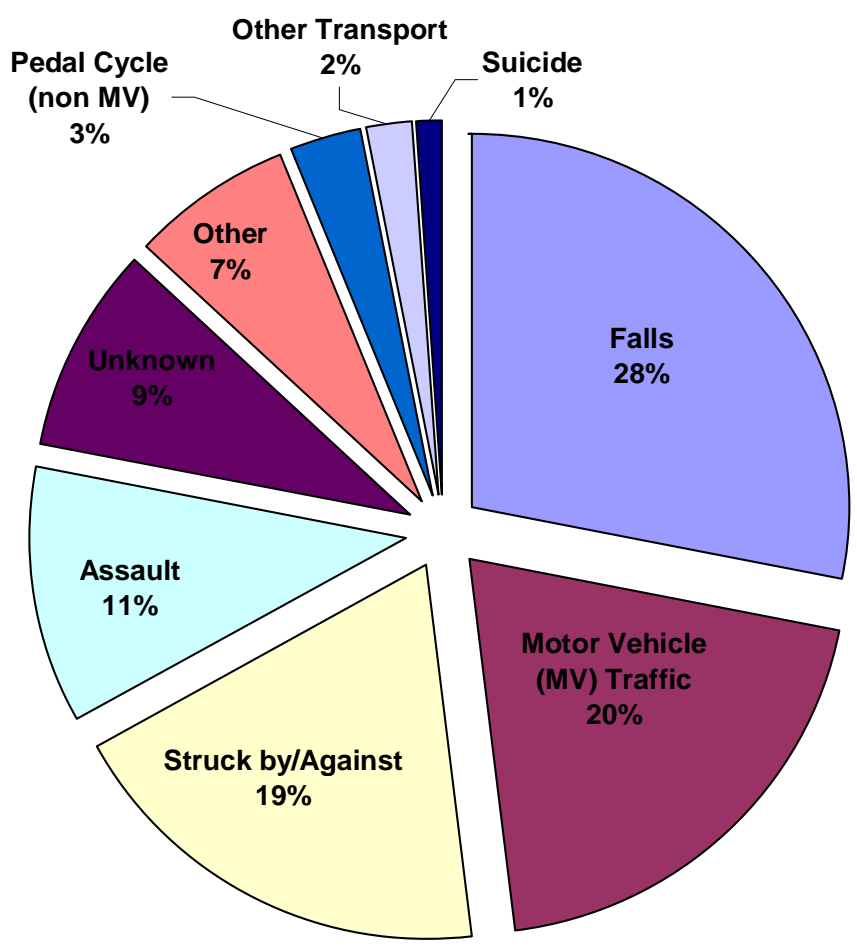

Source: Centers for Disease Control and Prevention, National Center for Injury Prevention and Control, Traumatic Brain Injury, http://www.cdc.gov/ncipc/tbi/Causes.htm.

\section{Symptoms}

At the time of injury, or some time after, TBI could be classified as mild, moderate, or severe.

- Mild TBI may cause a brief period of unconsciousness, mild confusion, or discomfort, while a more severe injury may cause longer periods of unconsciousness, nausea, vomiting, loss of coordination, or other symptoms. ${ }^{6}$

- Moderate TBI may be diagnosed when the patient experiences a loss of consciousness for less than 24 hours, and up to one week of post-traumatic amnesia.

- A TBI injury may be classified as "severe" if it involves more than one day of unconsciousness or more than one week of amnesia.

The perceived severity of the injury depends on a number of factors. Clinically, severity of TBI is measured by the Glasgow Coma Scale, ${ }^{7}$ level of unconsciousness, and the extent of posttraumatic amnesia. Physically, the patient may or may not experience structural abnormalities

\footnotetext{
${ }^{6}$ National Institute of Neurological Disorders and Stroke, NINDS Traumatic Brain Injury Information Page, http://www.ninds.nih.gov/disorders/tbi/tbi.htm.

${ }^{7}$ The Glasgow Coma Scale is a 15-point scale designed to assess the depth and duration of coma and impaired consciousness.
} 
such as skull fracture, bruising, or brain swelling. Trauma may be localized or diffuse, which can result in different symptoms.

In addition to physical symptoms, mental health diagnoses such as PTSD, depression, and anxiety are common for TBI patients, as is substance abuse. ${ }^{8}$ These symptoms may not develop until several months after a head injury. ${ }^{9}$ Emotional and behavioral changes are also common and can have lasting effects on interpersonal relationships. Recovery time varies, and some TBI patients have long-term physical effects, including epilepsy and an increased risk for conditions such as Alzheimer's disease, Parkinson's disease, and other neurological disorders that become more prevalent with age. ${ }^{10}$

\section{Treatment}

TBI is a complex injury with no specific standard of care or treatment regimen. Studies have shown that patients with mild TBI can make a complete recovery with little or no medical intervention. One study noted that patients with mild TBI recovered more quickly when provided with information on types of symptoms to expect. Other patients need more time and resources, and the nature of those needs varies on a case-by-case basis. ${ }^{11}$ In the civilian population, four types of outcomes have been identified for patients with moderate head injuries. Approximately $60 \%$ of patients with moderate head injuries make a positive recovery; an estimated $25 \%$ will be left with a moderate degree of disability; death or a persistent vegetative state will be the outcome in about $7 \%$ to $10 \%$ of cases; the remainder of patients will have a severe degree of disability. ${ }^{12}$

Individuals with TBI have diverse treatment needs; treatment ranges in complexity, depending on severity of the injury and the presence of co-conditions. Acute treatment of moderate or severe TBI includes immobilization of the patient to prevent further injury, and immediate treatment of acute physical symptoms. Once TBI patients leave the acute-care setting, some may benefit from a rehabilitation program. The National Institute for Neurological Disorders and Stroke (NINDS) recommends that TBI patients receive an individualized rehabilitation program based upon the patients' strengths and capacities. ${ }^{13}$ This program should be adapted over time. Prime candidates for rehabilitation are patients with less severe initial injuries, or those that have started to show significant improvement.

Management of patients with severe TBI requires a coordinated team of specialists, clinical assessments, imaging, and laboratory testing to facilitate recovery. To address both the physical

\footnotetext{
${ }^{8}$ T. Tanielian, L. Jaycox , ed., Invisible Wounds of War: Psychological and Cognitive Injuries, Their Consequences, and Services to Assist Recovery, Rand, 2008, pp. 126-127.

${ }^{9}$ American Association of Neurological Surgeons, “Traumatic Brain Injury,” 2007, http://www.aans.org/shared_pdfs/ Guidelines_Management_2007.pdf.

${ }^{10}$ National Institute of Neurological Disorders and Stroke, “Traumatic Brain Injury: Hope Through Research," National Institutes of Health, 2002, NIH Publication No. 02-158.

11 Veterans Health Administration, Polytrauma-Traumatic Brain Injury (TBI) System of Care, Department of Veterans Affairs, VHA Directive 2009-028, June 9, 2009, http://www1.va.gov/VHAPUBLICATIONS/ViewPublication.asp? pub_ID=2032.

${ }^{12}$ American Association of Neurological Surgeons, “Traumatic Brain Injury,” June 2006, http://www.neurosurgerytoday.org/what/patient_e/head.asp.

${ }^{13}$ M. Vital, Traumatic Brain Injury: Hope Through Research, National Institute of Neurological Disorders and Stroke, Bethesda, MD, September 2002, http://www.ninds.nih.gov/disorders/tbi/tbi_htr.pdf.
} 
and psychological effects of TBI, treatment and recovery is evaluated by assessments of brain function, functional independence, community participation, and self-reporting of well-being. For more severely injured patients, or for those whose recovery is slow, constant vigilance is required to prevent gradual onset of problems with joint mobility, respiratory status, and many other physiological functions. Patients with mild or moderate injuries, as well as severely injured patients who have improved sufficiently, may be candidates for outpatient therapy. According to the Centers for Disease Control and Prevention (CDC), treatment for individuals who have sustained a mild TBI may include increased rest, refraining from certain physical activities, management of symptoms, and education about what to expect during recovery.

\section{Care and Treatment of Veterans}

It has been estimated that as many as $20 \%$ of OEF/OIF veterans experience TBI. ${ }^{14}$ VA has taken steps to ensure that all veterans are screened for TBI, and that all veterans with TBI are diagnosed and treated. The following sections will describe servicemembers' care and treatment in the VA system, as well as challenges to care delivery for TBI, including the identification of TBI patients through screening and diagnosis, ensuring adequate care for veterans, and ensuring all veterans have access to that care.

As of January 2009, the VA system had screened 249,219 OEF/OIF veterans for TBI. Of those who were screened, approximately $20 \%(49,297)$ initially screened positive. Approximately 30,000 of those have completed their comprehensive evaluation, resulting in $13,726(5.3 \%$ of all the veterans screened) confirmed TBI diagnoses. ${ }^{15}$ The remaining cases are either waiting to be screened, or have refused further treatment. In comparison to other estimates, this number may seem low. However, this number does not reflect the true prevalence of TBI in the OEF/OIF veteran population for several reasons. Some who screened positive in the initial screen currently are waiting for a comprehensive evaluation, and a small number have chosen not to undergo more testing for TBI. Some servicemembers, particularly those with moderate to severe TBI, are diagnosed by DOD while still on active duty. Additionally, less than half of discharged servicemembers have enrolled in the VA health system and may receive healthcare, as described below.

VA provides a range of services to address the needs of veterans with TBI, including outreach and education, and provides benefits enrollment information to all veterans. For those who are eligible, and who choose to enroll, VA provides screening, diagnosis, treatment, and care coordination services for the veterans, and support services for their families. Veterans generally must enroll in the VA health care system to receive medical care. Eligibility for enrollment is based primarily on previous military service, disability, and income. VA provides free inpatient and outpatient medical care to veterans for service-connected conditions, and to low-income veterans for non-service-connected conditions. ${ }^{16,17}$ Additionally, VA has encouraged OEF/OIF

\footnotetext{
${ }^{14}$ T. Tanielian, L. Jaycox , ed., Invisible Wounds of War: Psychological and Cognitive Injuries, Their Consequences, and Services to Assist Recovery, Rand, 2008.

${ }^{15}$ Congressional Research Service inquiry to VHA Program Office, January 21, 2009.

${ }^{16}$ The term "service-connected" means, with respect to disability, that such disability was incurred or aggravated in the line of duty in the active military, naval, or air service. VA determines whether veterans have service-connected disabilities, and for those with such disabilities, assigns ratings from 0 to $100 \%$ based on the severity of the disability. Percentages are assigned in increments of $10 \%$.
} 
veterans to enroll in health services by offering a five-year post-discharge automatic enrollment period for health care. Veterans who enroll during this period are provided care, regardless of service-connected disability status.

\section{Screening}

Servicemembers are screened by DOD at discharge and six months following discharge. Following discharge from active duty, a self-administered post-deployment health reassessment (PDHRA) survey is administered by DOD to all servicemembers. This survey includes questions about mental health and TBI symptoms, among other health conditions. However, DOD postdeployment discharge screening of servicemembers may not identify individuals with latent symptoms of TBI. ${ }^{18}$ Servicemembers are also screened if they choose to enroll in VA services, and have not previously been diagnosed with a TBI. This section outlines the TBI screening process for veterans.

In April 2007, the VA implemented a policy requiring TBI screening for all OEF/OIF veterans who had not previously been diagnosed with TBI. Prior to that date, TBI screening was not required. The policy coincided with the implementation of a computer-based screening tool with an automated reminder at primary care, dental, and urgent care visits to identify patients at risk for TBI. This reminder, together with reminders for screens for PTSD, depression, alcohol abuse, and infectious diseases endemic to Southwest Asia, constitutes the "Afghan and Iraq PostDeployment Screen."19

Eligible veterans who choose to enroll in the VA Healthcare System are screened for TBI at their initial appointment in the VA system. The screening tool consists of four sequential sections: (1) events, (2) immediate symptoms following events, (3) new or worsening symptoms since events, and (4) current symptoms. The questions in each of these sections are designed to identify exposure to events that increase the risk of TBI. Positive responses at one level open the next section, and negative responses end the screen. If a veteran answers "yes" to one or more questions in each of the four sections, the screen is considered positive, and the veteran is referred for follow-up testing. The follow-up evaluation with a specialist will determine whether the veteran has a TBI or not.

The screening tool currently employed by VA is based on a modified version of the Brief Traumatic Brain Injury Screen (BTBIS), a TBI screening tool used by DOD. Prior to implementation of the modified screening tool, VA did not assess its clinical validity and reliability, which raised questions about its effectiveness. ${ }^{20}$ The Government Accountability Office (GAO) raised this issue in a 2008 report on VA screening and evaluation of TBI. ${ }^{21}$ In its

\footnotetext{
(...continued)

${ }^{17}$ CRS Report R40737, Veterans Medical Care: FY2010 Appropriations, by Sidath Viranga Panangala.

${ }^{18}$ T. Tanielian, L. Jaycox , ed., Invisible Wounds of War: Psychological and Cognitive Injuries, Their Consequences, and Services to Assist Recovery, Rand, 2008.

${ }^{19}$ Veterans Health Administration, Implementation of the National Clinical Reminder for Afghan and Iraq PostDeployment Screening, Department of Veterans Affairs, VHA Directive 2005-055, Washington, DC, December 1, 2005.

${ }^{20}$ K.F. Carlson et al., "PTSD and Other Psychiatric Comorbidities in OEF/OIF VA Users with TBI," HSR\&D Conference Presentation, Baltimore, MD, February 2009.

${ }^{21}$ Congress has mandated in the National Defense Authorization Act for Fiscal Year 2008 that VA and DOD establish (continued...)
} 
findings, GAO noted several issues with implementation of the automated reminder and screening tool. Among the issues were a lack of staff training on the tool, and the use of a screening that was modified from DOD's version, but not re-evaluated for clinical validity and reliability. GAO recommended that VA conduct research to assess the screening tool. VA has since undertaken several studies to assess the validity and implementation of the tool. ${ }^{22}$

\section{Diagnosis}

For veterans who may have a TBI, but were not diagnosed while on active duty, screening is the first step toward obtaining treatment. However, a positive screen does not conclusively diagnose TBI. The screening tool is designed to be sensitive, to catch all potential cases. Of the nearly 50,000 veterans with a positive screen for TBI, 30,000 had completed their follow-up comprehensive examination as of January 2009, and fewer than 50\% of those have been diagnosed with a TBI. ${ }^{23}$ Veterans with a positive screen are offered a referral for a comprehensive follow-up evaluation and services with a specialty provider who is qualified to determine a TBI diagnosis.

The delayed onset of symptoms in combination with difficulty navigating the system due to mental health issues may prevent some veterans from seeking treatment. Co-occurrence of psychological symptoms can also complicate diagnosis and treatment. PTSD and TBI have several overlapping symptoms, including insomnia, memory problems, depression, and anxiety, which complicates differential diagnosis and treatment. One study found that $85 \%$ of OEF/OIF veterans with TBI had psychiatric comorbidities. PTSD was the most common condition, followed by substance use disorders $(26 \%)$ and major depressive disorders $(19 \%) .{ }^{24}$ In another study, the presence of PTSD in patients with TBI was very strongly indicated by intrusive memories, nightmares, or emotional reactivity. ${ }^{25}$ To address the issue of determining the appropriate diagnosis, VA has initiated the Markers for the Identification, Norming, and Differentiation of TBI and PTSD (MIND) study, which will help determine consistent differential diagnostic criteria for TBI and PTSD.

\section{Treatment and Rehabilitation}

Servicemembers who sustain injuries receive immediate care on the battlefield, and are then transported to a military medical hospital for treatment. Battlefield treatment may include the removal of foreign bodies, control of bleeding, or craniotomy to relieve pressure from swelling. Once stabilized, DOD can elect to send injured servicemembers with TBI or other complex

\footnotetext{
(...continued)

a joint interagency program office to act as a single point of accountability in the development of electronic health records systems or capabilities that allow for full interoperability (generally, the ability of systems to exchange data) by September 30, 2009.

${ }^{22}$ Department of Veterans Affairs, FY2010 Congressional Budget Submission, p. 87, http://www4.va.gov/budget/ summary/2010/Volume_1-Summary_Volume.pdf.

${ }^{23}$ Congressional inquiry to VHA Program Office, January 21, 2009.

${ }^{24}$ K.F. Carlson, D.B. Nelson, and S.M. Nugent et al., "PTSD and Other Psychiatric Comorbidities in OEF/OIF VA Users with TBI," HSR\&D 2009 National Meeting, 2009.

${ }^{25}$ R. Bryant et al., "Post Traumatic Stress Disorder After Severe Traumatic Brain Injury," Am J Psychiatry, vol. 157, no. 4 (April 2000).
} 
trauma to VA medical facilities for medical and rehabilitative care. The following sections describe treatment and rehabilitation in the VA system, including a description of ongoing research in these areas.

Many servicemembers who sustain TBI are treated and return to active duty, home, and social responsibilities. Most mild cases of TBI can be resolved without medical attention and may be best served by receiving educational materials. ${ }^{26}$ Others may need ongoing treatment and rehabilitation. Outside of the system, VA must work with DOD and other care delivery systems to ensure communication of all relevant information during the transition of care from one entity to the other.

Regardless of the setting, most rehabilitation centers emphasize compensatory strategies, which essentially help patients learn to reach the maximum level of function allowed by their impairments. The concept of cognitive retraining, which presumes that at least some of the brain's cognitive capacity can be restored by constant repetition of certain simple tasks, is emphasized in rehabilitation. Another major goal of TBI rehabilitation is working with patients' families to educate them about behavior they can realistically expect and how they can best help their injured family member. Veterans' care is integrated and coordinated through the Polytrauma System of Care.

\section{Polytrauma System of Care}

Many servicemembers are eventually referred to one of the Defense and Veterans Brain Injury Centers (DVBIC), or to a VA Polytrauma Center. The VHA defines polytrauma as "injuries to two or more physical regions or organ systems which occur as a result of the same incident and cause physical, cognitive, psychological, or psychosocial impairments and functional disability." As such, the VA polytrauma system of care is designed to address the multiple types of injuries that occur in conjunction with TBI.

In 2004, Congress passed the Veterans Health Programs Improvement Act. This act directed VA to designate cooperative centers for care of TBI and polytrauma associated with combat injuries. In response, VA introduced the TBI/Polytrauma System of Care (PSC) in April 2005. ${ }^{27}$ This integrated, nationwide system encompasses over 100 VA facilities dedicated to serving the needs of servicemembers with TBI and polytrauma. The PSC operates geographically as a "hub and spoke" model with four tiered levels of care. The system is designed to be geographically dispersed, thereby making the specialized treatment requirements of veterans with TBI more accessible.

\footnotetext{
${ }^{26}$ C.W. Hoge, J. McGurk, and A.L. Thomas et al., "Mild Traumatic Brain Injury in US Soldiers Returning from Iraq," New England Journal of Medicine, vol. 358, no. 5 (2008), pp. 453-463.

${ }^{27}$ Veterans Health Administration, Polytrauma-Traumatic Brain Injury (TBI) System of Care, Department of Veterans Affairs, VHA Directive 2009-028, Washington, DC, June 9, 2009.
} 
Figure 2.VA Polytrauma System of Care

Location of Polytrauma Rehabilitation Centers and Polytrauma Network Sites

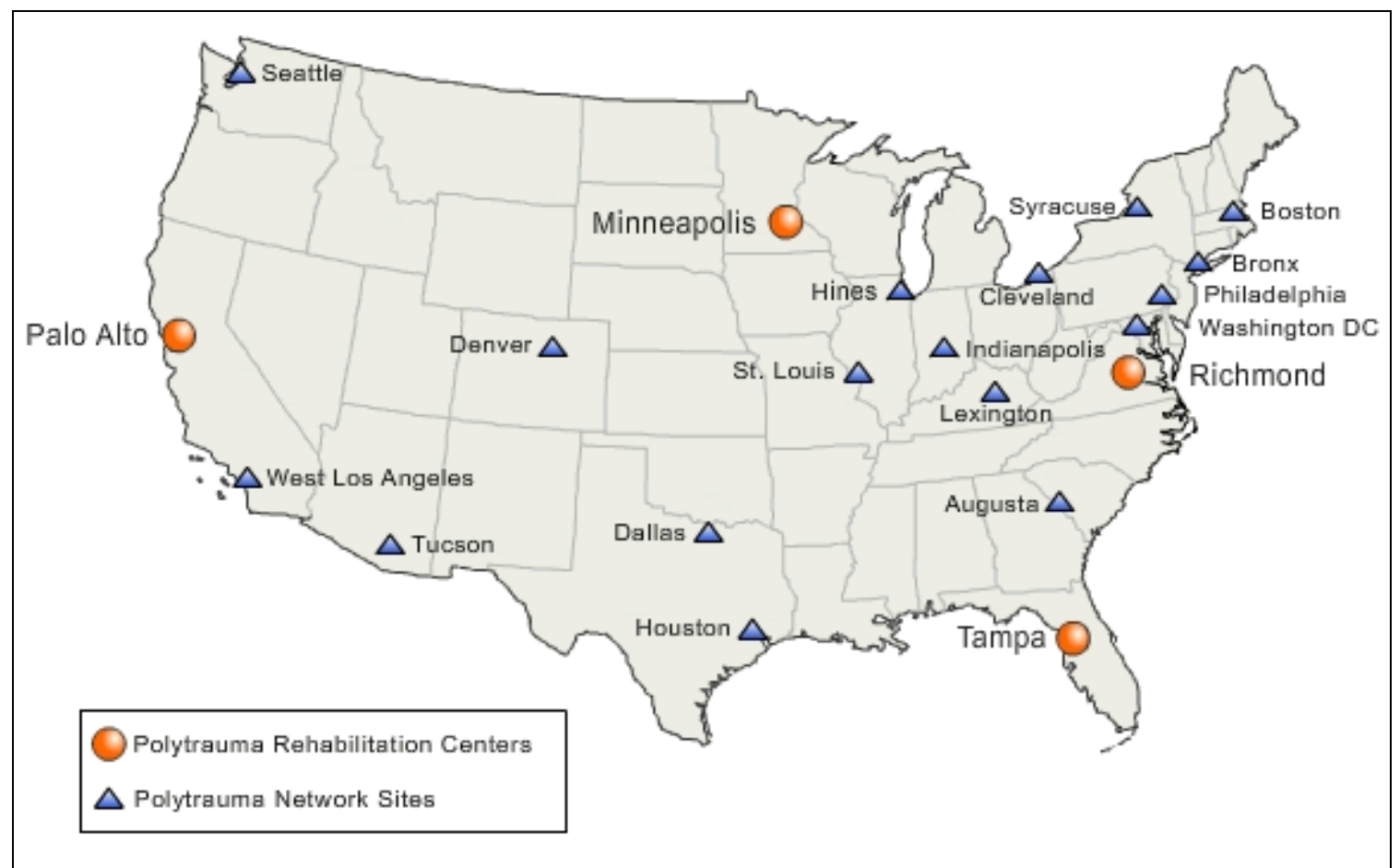

Source: U.S. Department of Veterans Affairs.

Note: A fifth Polytrauma Rehabilitation Center will be located in San Antonio, TX, upon completion.

VA recommends that all veterans experiencing a polytraumatic injury be referred to one of the four ${ }^{28}$ Polytrauma Rehabilitation Centers (PRC), the "hubs" of the system. These centers provide acute care for eligible veterans who have sustained severe disabling injuries including, but not limited to, TBI, amputation, visual and hearing impairment, spinal cord injury, musculoskeletal injuries, wounds, and psychological trauma. PRCs provide specific inpatient, transitional, and outpatient rehabilitation tailored to individual patterns of impairments. These centers provide high-intensity care in a residential setting while optimizing the patient's medical condition and improving basic functioning. The interdisciplinary rehabilitation teams include a physiatrist, rehabilitation nurses, occupational and physical therapists, neuropsychologists, recreation therapists, speech-language pathologists, social workers, and vocational counselors.

The PRC provides clinical case management of referrals prior to admission and follow-up case management following discharge. The clinical case manager monitors each patient's progress on medical and functional problems, supports coordination of ongoing rehabilitation care, advocates for the patient's needs, and makes recommendations for alternative care settings when appropriate. Patients are eventually dispersed to the "spoke" facilities, which offer post-acute care, rehabilitation, and care coordination.

Over time, as the patient needs less acute, specialized, or intensive levels of care, VA has tried to make that care more accessible. Polytrauma network sites (PNS), which are located in each of

${ }^{28}$ A fifth center is currently in the design phase. 
VA's 21 regional health care networks, provide specialized, post-acute rehabilitation for patients who need less intensive treatment, over a longer period of time. They also offer outpatient therapy and day treatment. The next level of care, Polytrauma Clinic Support Teams (PCST), provide facility-based teams with rehabilitation expertise that offer coordination of care, group therapy, and day treatment programs. These therapies address ongoing functional impairments and the management of stable polytrauma conditions.

Every VA facility that is not otherwise designated as a part of the polytrauma system of care has a Polytrauma Point of Contact (PPOC) who is responsible for coordinating the treatment of veterans at their facility. The role of the PPOC is to ensure that veterans are referred to a facility capable of providing the services they require. At all levels of the PSC, the presence of a mental health care professional can be helpful in addressing mental health issues as they arise. VA care of TBI and co-occurring mental health conditions, including PTSD, focuses on reducing functional disability.

\section{Coordination of Treatment}

Within the VA, patient care for TBI is tracked through a web-based application that monitors each individual who is screened and referred for comprehensive evaluation and follow-up care. This tracking application is used nationally to capture data on patients who have screened positive for possible TBI, those referred for follow-up evaluation, those who have completed the TBI evaluation, and actions taken to reach those who have not completed the evaluations. The VA creates and distributes monthly reports to assist facilities in developing and refining their clinical processes.

For servicemembers who have been previously diagnosed with a TBI, there may be delays in the transition of medical records from the DOD system to VA due to an identified lack of interoperability of electronic health records between the two agencies. ${ }^{29}$ Servicemembers who were diagnosed with TBI while on active duty, in most cases those with moderate or severe TBI, will need coordination of care between DOD and VA. Although progress has been made in this area, it is an ongoing challenge. ${ }^{30}$

The National Defense Authorization Act (NDAA) of 2008 (P.L. 110-181) included provisions known as the Wounded Warrior Act. These provisions were drafted to address congressional concerns about the quality and availability of medical, dental, and mental health services for OEF/OIF servicemembers and veterans. ${ }^{31}$ This act required DOD and VA to coordinate research and treatment of TBI and other conditions, and to plan for and evaluate the seamless transition of servicemembers to the veterans health care system. This act also required the VA to establish a rehabilitation and reintegration plan for every servicemember or veteran who receives care for TBI, based on a comprehensive assessment of physical, cognitive, vocational, neuropsychological, and social impairments. Each veteran is assigned a case manager who is

\footnotetext{
${ }^{29}$ U.S. Congress, House Committee on Veterans' Affairs, Program Office Improvements Needed to Strengthen Management of VA and DoD Efforts to Achieve Full Interoperability, Electronic Health Records, $111^{\text {th }}$ Cong., $1^{\text {st }}$ sess., July 14, 2009.

${ }^{30}$ T. Tanielian, L. Jaycox, ed., Invisible Wounds of War: Psychological and Cognitive Injuries, Their Consequences, and Services to Assist Recovery, Rand, 2008.

${ }^{31}$ CRS Report RL34371, “Wounded Warrior" and Veterans Provisions in the FY2008 National Defense Authorization Act.
} 
required to facilitate implementation of each plan and coordination of care with family members and/or legal guardians involved in developing the plan as much as possible. VA was also authorized through this act to utilize non-VA facilities to deliver the necessary care for servicemembers and veterans with TBI. The act also required the Secretaries of DOD and VA to ensure that TBI victims receive a TBI-specific medical designation, rather than a generic disease classification. This issue was addressed with a revision of diagnostic codes, which are currently being implemented.

\section{Reintegration}

Servicemembers with TBI may experience personality and emotional changes after the injury, which may impact their reintegration into family and community life. They experience difficulty with problem-solving, recalling information, and multi-tasking. ${ }^{32}$ VA researchers are concentrating on treatment that will improve overall integration and quality of life for veterans with TBI ${ }^{33}$ Researchers have also developed a project exploring community reintegration for servicemembers with TBI to promote seamless transition for those currently being treated, and those who will be treated in the future. Researchers are also assessing which available resources work best to reduce caregiver strains.

Some veterans may qualify for the Federal Recovery Coordinator Program. This program was established in 2008 to address the needs of severely injured veterans who may need additional assistance to obtain the needed level of care. ${ }^{34}$ Federal recovery coordinators track the care, management, and transition of recovering servicemembers and veterans through recovery, rehabilitation, and reintegration. This is achieved by coordinating federal health care teams and private community resources to achieve the personal and professional goals of qualifying servicemembers and veterans. Coordinators link servicemembers and veterans with public and private resources, according to their needs. They are assigned to active-duty servicemembers, and continue to assist veterans during and after their transition to civilian life.

\section{Research}

The Defense and Veterans Brain Injury Center (DVBIC), a congressionally mandated collaboration of DOD and VA, conducts clinical research on TBI. The DVBIC is composed of a multi-site network, including a growing number of DOD and VA hospitals as well as civilian TBI rehabilitation programs; each site works collaboratively to provide and improve TBI care for active duty military, veterans, and their eligible beneficiaries. DVBIC is currently researching a number of topics, including substance abuse and TBI, a prevalence study of mild TBI, and several studies on neuropsychological function after a TBI.

The 2008 National Defense Authorization Act required VA to establish and maintain a Traumatic Brain Injury Veterans Health Registry, including information on all OEF/OIF servicemembers

\footnotetext{
${ }^{32}$ S.S. Dikmen, J.E. Machamer, and H.R. Winn et al., "Neuropsychological Outcome at 1-Year Post Head Injury," Neuropsychology, vol. 9 (1995), pp. 80-90.

${ }^{33}$ R. Vanderploeg, K. Schwab, and W. Walker et al., "Rehabilitation of Traumatic Brain Injury in Active Duty Military Personnel and Veterans: Defense and Veterans Brain Injury Center Randomized Controlled Trial of Two Rehabilitation Approaches," Arch Phys Med Rehabil, vol. 89 (December 2008), pp. 2227-2238.

${ }^{34}$ Department of Veterans Affairs, "VA-DoD Program Serves Severely Disabled Combat Veterans," press release, May 7, 2008, http://www1.va.gov/opa/pressrel/pressrelease.cfm?id=1499.
} 
who exhibit TBI symptoms, and who apply for care or compensation from VA on the basis of any disability. VA is currently partnering with the National Institute of Disability and Rehabilitation Research (NIDRR) to develop this registry. This registry will facilitate future research by providing longitudinal data on the demographics, military service data, injury information, and treatment of all veterans with TBI. Under the Wounded Warrior Act, VA was also required to implement a pilot program to assess the effectiveness of providing assisted-living services to eligible veterans. The pilot program is ongoing at this time.

TBI is one of the research priority areas for VA scientists. The FY2010 President's Budget request to Congress for VA includes \$298 million for TBI research for all veterans, with an additional request for $\$ 63$ million specifically for OEF/OIF veterans. ${ }^{35}$

\section{Access to Care}

Forty-seven percent of veterans from these conflicts have enrolled in the VA system through the third quarter of FY2009. ${ }^{36}$ While this enrollment rate of veterans may seem low, it has been increasing since the extension of benefits to five years post-discharge from the military. Additionally, veterans who do not enroll in the VHA system may have health coverage from other sources, such as TRICARE or private insurance. Some who do not receive care may not perceive the need for care due to mild TBI and other, less obvious injuries, including mental illnesses such as PTSD. For others, there may be different barriers to accessing care. Returning servicemembers may not be aware of the symptoms of mild TBI, or the treatments available through the VA. Others may suspect a TBI and/or mental illness, but may avoid diagnosis and treatment due to cultural perceptions of mental illness and potential impact on future employment or enrollment in the Reserves or National Guard. Veterans may be readjusting to life at home after a deployment, and a TBI diagnosis could cause further disruption, including the time and effort required to access treatment, particularly if treatment is not readily available. These potential barriers to accessing TBI diagnosis and treatment are discussed below.

\section{Physical, Financial, and Other Barriers to Accessing Care}

To address the issue of low enrollment, and the concern of potential undiagnosed illness due to non-enrollment in VA services, VA has increased outreach efforts to increase awareness of, and participation in, TBI screening of OEF/OIF veterans. The VA provides outreach to veterans during the post-deployment health reassessments (PDHRA) administered by DOD $^{37}$ in communities, including DOD post-deployment events, at military treatment facilities and at National Guard and Reserve facilities. Several treatment facilities have contacted veterans to offer screening to those who received care prior to implementation of the VA's screening tool. In these outreach settings, VA staff educate veterans about TBI screening and other benefits.

\footnotetext{
${ }^{35}$ VA Department of Veterans Affairs, FY2010 Budget Submission, Medical Programs and Information Technology Programs, vol. 2 of 4, May 2009.

${ }^{36}$ VA Office of Public Health and Environmental Hazards, Analysis of VA Health Care Utilization Among US Global War on Terrorism (GWOT) Veterans, Department of Veterans Affairs, Washington, DC, October 2009.

${ }^{37}$ Following discharge from active duty, a self-administered post-deployment health reassessment (PDHRA) survey is administered by the Department of Defense (DOD) to all servicemembers. This survey includes questions about mental health and TBI symptoms, among other health conditions.
} 
Veterans may have difficulty keeping follow-up appointments due to work and family commitments, or the lack of nearby treatment facilities. ${ }^{38}$ VA has tried to address this challenge through the polytrauma system of care, but some veterans continue to experience barriers to care access. The PSC is designed to be geographically dispersed; however, some have identified ongoing gaps in access to treatment for rural veterans. Some veterans who are recovering from moderate to severe TBI may be best served in a home or residential setting. VA Home Care and Health Services offer in-home rehabilitation on a limited basis for certain veterans who cannot access a treatment center. However, access to local and specialized therapies remains limited. Congress has addressed this issue by providing training and support for family caregivers. Legislation has been introduced in the $111^{\text {th }}$ Congress to further address caregiver support, including training and respite care.

Adequate staffing capacity is necessary to provide an appropriate level of care for veterans with TBI. In the clinical setting, GAO has identified that a shortage of trained staff affects the provision of the recommended standard of care for potential TBI patients. ${ }^{39}$ VA clinical providers who administer the TBI screening tool must complete a TBI training module. Some providers who were administering the tool had not completed the required training. One concern raised in the GAO study was a lack of clinical staff to administer the TBI screening tool, and a need for further training on the tool. In response to these findings, VA implemented a TBI screening performance measure beginning in FY2008 to assess the extent of TBI screening for OEF/OIF veterans.

\section{Sociopsychological Barriers}

TBI patients exhibit a variety of cognitive and emotional impairments, including attention deficits, interpersonal difficulties, and apathy. Some servicemembers may not be fully aware of behavior changes that result from their injuries. In one study, servicemembers recovering from moderate to severe TBI tended to rate their functioning in these areas higher than close family members rated them, when asked. ${ }^{40}$ This lack of awareness of problems can make family support of injured servicemembers even more challenging; the lack of recognition of problems can impede their willingness to get treatment. Additionally, these impairments may compromise servicemembers' capacity to seek care on their own. ${ }^{41}$

Injured servicemembers with cognitive or emotional impairments may have trouble navigating the system that is designed to help them. Veterans with TBI may be eligible for the Federal Recovery Coordinator Program, which helps severely injured veterans navigate the treatment process. Finally, returning servicemembers have work and family commitments to readjust to

\footnotetext{
${ }^{38}$ T. Tanielan, L. Jaycox, ed., Invisible Wounds of War: Psychological and Cognitive Injuries, Their Consequences, and Services to Assist Recovery, RAND Center for Military Health Policy Research, Santa Monica, CA, 2008, pp 307326.

${ }^{39}$ U.S. Government Accountability Office, Mild Traumatic Brain Injury Screening and Evaluation Implemented for OEF/OIF Veterans, but Challenges Remain, 08-276, February 2008.

${ }^{40}$ R. Vanderploeg, H. Belanger, and J. Duchnick et al., "Awareness Problems Following Moderate to Severe Traumatic Brain Injury: Prevalence, Assessment Methods, and Injury Correlates," JRRD, vol. 44, no. 7 (2007), pp. 937-950.

${ }^{41}$ H.L. Lew et al., "Persistent Problems after Traumatic Brain Injury: The Need for Long-Term Follow-up and Coordinated Care," JRRD, vol. 43 (2006), pp. vii-x.
} 
after being gone several months. It has been speculated that soldiers leaving war zones may minimize or downplay mental health symptoms to avoid any delay in their return home. ${ }^{42}$

Additionally, veterans may not be signing up for VA care because a TBI diagnosis is closely associated with mental illness. According to GAO, there is an ongoing perception that being diagnosed with a TBI would affect veterans' ability to stay in the National Guard or Reserves, or affect other future employment plans. ${ }^{43}$ Servicemembers may fear that a TBI is closely associated with a mental health diagnosis, and this could have an adverse effect on future employment, both within and outside of the military.

Finally, there is a perception among OEF/OIF veterans that VA is for the older generations of veterans, and not for veterans of the current conflicts. VA has tried to address this perception through outreach and adaptation of VA services to the needs of recent veterans.

\section{Issues for Congress}

As veterans return home from these conflicts, VA has faced an increasing demand for its services, particularly for treatment of "signature injuries," such as TBI. The National Defense Authorization Act (NDAA) of 2008 included provisions known as the Wounded Warrior Act. ${ }^{44}$ These provisions addressed congressional concerns about the quality and availability of medical, dental, and mental health services for OEF/OIF servicemembers and veterans. Federal policymakers continue to consider policies and proposed legislative initiatives to address TBI among the veteran population. This section briefly describes policies that Congress may consider regarding support and care of veterans with traumatic brain injury. They are organized into the following topics: (1) identification and screening, (2) continuity of care, (3) staffing and access to care, (4) social and psychological issues, and (5) research.

\section{Identification and Screening}

Policymakers have considered whether the current screening and outreach efforts are effective and adequate. Some have raised concerns that VA is not reaching all former servicemembers who may have TBI due to the 2007 implementation of universal TBI screening in the VA system. VA has addressed this concern by initiating a program to contact all OEF/OIF veterans by telephone to inform them of the medical services and other benefits available to them through the VA. The first phase of this program targeted veterans who were sick or injured while in Iraq or Afghanistan, while the second will target all others who were discharged from active duty but who have not contacted VA for services.

Policymakers have also proposed initiatives that would require VA and DOD to coordinate screening and treatment of servicemembers. Some have expressed concern that veterans with mild TBI, in particular, may have difficulty navigating the process of obtaining the appropriate

\footnotetext{
${ }^{42}$ T. Tanielan, L. Jaycox, ed, Invisible Wounds of War: Psychological and Cognitive Injuries, Their Consequences, and Services to Assist Recovery, RAND Center for Military Health Policy Research, Santa Monica, CA, 2008, p. 7.

${ }^{43}$ U.S. Government Accountability Office, Mild Traumatic Brain Injury Screening and Evaluation Implemented for OEF/OIF Veterans, but Challenges Remain, 08-276, February 2008.

${ }^{44}$ CRS Report RL34371, "Wounded Warrior" and Veterans Provisions in the FY2008 National Defense Authorization Act, by Sarah A. Lister, Christine Scott, and Sidath Viranga Panangala.
} 
disability status to get treatment, or transitioning from one care system to the other. Programs to ensure the availability of case managers and coordinators have been created for veterans, and some suggest these programs need to be strengthened or expanded.

\section{Continuity of Care}

Policymakers and others have expressed concern over the lack of communication of timely and accurate medical information between DOD and VA. The NDAA required DOD and VA to coordinate research and treatment of TBI and other conditions, and to plan for and evaluate the seamless transition of servicemembers to veteran health care systems. This collaboration is ongoing. Policymakers have addressed and continue to assess the ongoing need for VA and DOD to coordinate a continuum of mental health and TBI care for veterans. Congress has proposed legislation to establish a committee that will continually assess VA care for veterans with TBI.

\section{Staffing and Access to Care}

The FY2010 President's Budget Request ${ }^{45}$ includes assurances that VA is working to fund programs that improve veterans' access to mental health services across the country, including those who suffer from PTSD and TBI as a result of their service in OEF/OIF. Congress has considered legislation that would address the ongoing concerns of TBI treatment and access to care. Many of the measures introduced include funding for PTSD and other combat-related mental health issues, in addition to funding for TBI programs. Some have proposed expanding traumatic injury insurance coverage and benefits to non-OEF/OIF veterans, requiring remote mental health and TBI assessments for veterans without current access to those services through the VA, maintaining benefits for survivors of service members with PTSD or TBI who commit suicide, and providing family caregiver training and support, including respite care.

Congress and VA have considered legislation that would establish pilot programs for servicemembers with TBI to address access issues, including a program to provide training and certification for family caregivers as personal care attendants. Another proposed pilot program would provide respite care by students in graduate programs related to mental health or rehabilitation, and also provide specialized residential care and rehabilitation services for certain $\mathrm{OEF} / \mathrm{OIF}$ veterans. Other proposals to expand care include further authorization of TBI rehabilitation at non-VA facilities, and the expansion of tele-health alternatives for veterans with TBI and PTSD who live in rural areas. Other proposals would provide training, certification, and support for family caregivers of seriously wounded OEF/OIF veterans, including those with TBI or psychological trauma.

\section{Social and Psychological Issues}

Congress has proposed various initiatives for routine TBI and mental health screening for servicemembers, pre- and post-deployment. Congress may review the role of VA and other agencies in servicemember reintegration into work and family life to ensure that social stigma does not provide a barrier to recovery. Other proposed congressional initiatives address potential

${ }^{45}$ VA Department of Veterans Affairs, FY2010 Budget Submission, Medical Programs and Information Technology Programs, vol. 2 of 4, May 2009. 
suicide risks for veterans from $\mathrm{OEF} / \mathrm{OIF}$, and address coverage for survivors of suicide victims with combat-related PTSD, TBI, or other mental health issues.

\section{Research}

Finally, policymakers have supported increased funding for TBI treatment and research. In FY2009, VA funding for TBI research for all veterans increased by $17 \%$ over the previous year; additionally, funding for TBI research for OEF/OIF veterans in FY2009 was increased by 38\% over the previous year. Congress has asked VA to conduct research on the following: mild to severe forms of TBI; visually related neurological conditions; means of improving the diagnosis, rehabilitative treatment, and prevention of TBI; and dual diagnosis of PTSD and TBI, and other conditions.

\section{Author Contact Information}

Amalia K. Corby-Edwards

Analyst in Public Health and Epidemiology

acorbyedwards@crs.loc.gov, 7-0423 